\title{
Towards the standardization of ballistocardiography systems for J-peak timing measurement
}

\author{
Joan Gomez-Clapers ${ }^{1}$, Albert Serra-Rocamora ${ }^{1}$, Ramon Casanella ${ }^{1}$, Ramon Pallas-Areny ${ }^{1}$ \\ ${ }^{I}$ Instrumentation, Sensors and Interfaces Group, Universitat Politècnica de Catalunya - BarcelonaTech \\ Email:joan.gomez-clapers@upc.edu
}

\begin{abstract}
There is a growing interest in accurately measuring the timing of the $\mathrm{J}$ peak of the ballistocardiogram (BCG) in order to obtain cardiovascular function markers non-invasively, especially in modern home healthcare applications. In this paper we have studied the effect that some common uncertainty sources have in the time measurement of the J peak. This is a necessary step towards the standardization of modern ballistocardiography systems equivalent to that available for EGC systems. We conclude that, to reduce J peak time uncertainty below the measured intrinsic uncertainty of about $\pm 2 \mathrm{~ms}$, the minimal bandwidth should be from $1.5 \mathrm{~Hz}$ to $22.5 \mathrm{~Hz}$; the sampling frequency can be decreased up to $50 \mathrm{~Hz}$ when using cubic spline interpolation; 5 bits are required to quantify the signal, and signal-to-noise ratio (SNR) and signal-to-interference ratio (SIR) should be over $40 \mathrm{~dB}$ and $3 \mathrm{~dB}$ respectively.
\end{abstract}

Keywords: Ballistocardiogram, acquisition system, biomedical instrumentation, standardization.

\section{Introduction}

Prevention and early detection can lead to a decrease in incidence of many diseases but current healthcare services are not meant for large population screenings. In order to reduce hospital occupancy and diminish the workload of medical staff, some diagnoses could be performed at home, in secondary medical facilities or even in pharmacies [1]. Two options that have been widely explored in home healthcare are to measure biosignals from sensors embedded in furniture such as beds or chairs and to improve the capabilities of existing household devices such as bathroom scales. Most of these new measurement systems are designed for simplicity of use, comfort and cost-effectiveness, which are usually achieved by measuring physiological electrical or mechanical signals.

In this context, the ballistocardiogram (BCG), initially understood as the recording of body movements caused by cardiovascular activity [2], has gained a renewed interest [3] because forces related to those movements can be measured from the strain gages in common electronic bathroom scales $[4,5,6]$ or other sensors placed in chairs [7] or beds [8]. Although the BCG can seemingly provide valuable information about the state of the cardiovascular system $[2,3,9]$, until now the signal has been mostly proposed to monitor heart rate [4] and sleep stages [8].

Nevertheless, several recent efforts in this field target the measurement of the delay of the J peak of the BCG acquired from weighing scales with respect to the R peak of the electrocardiogram (ECG), the so-called RJ interval. This interval represents the time from the electrical activation of the ventricles to the greatest vertical force derived from the cardiovascular activity and has been proposed to track hemodynamic changes [5], beat-tobeat blood pressure changes [6] and cardiac contractility [10]. Figure 1 shows a sample of ECG and BCG for a single cardiac cycle where these two prominent waves and other ECG and BCG waves are identified.

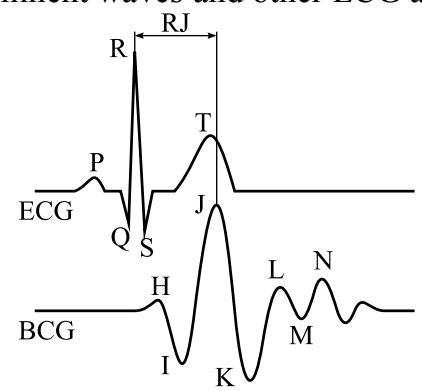

Figure 1. Single-beat ECG (top) and BCG (bottom) samples with their main waves and the RJ interval.

According to the bibliography, typical RJ durations in resting conditions range from $180 \mathrm{~ms}$ to $240 \mathrm{~ms}$ and under large hemodynamic changes induced by maneuvers such as Valsalva's $[5,6,10]$ or paced respiration [11] they may reach from $150 \mathrm{~ms}$ to $300 \mathrm{~ms}$. RJ recordings during experiments that involve maneuvers usually show a variability which frequency is above $0.5 \mathrm{~Hz}$, hence higher than that of changes induced by maneuvers. Those 
high-frequency changes in RJ intervals constitute an inherent uncertainty, in the sense defined in [12], and must be accounted for when RJ intervals are measured to assess hemodynamic changes.

Further, the possible effect of the data acquisition system on the measurement result should be lower than that inherent RJ uncertainty. For the ECG, system specifications to reliably monitor heart rhythm from R peak detection were analyzed and defined long ago $[13,14]$. Nevertheless, this is not the case of the J peak, for which the influence of sources of uncertainty already considered in the detection of the R peak of the ECG, such as the minimal system bandwidth as well as others that can introduce significant systematic and random timing displacements [15] are still to be analyzed.

With regard to system bandwidth, some high-pass cutoff frequencies for BCG found in the published work are $0.1 \mathrm{~Hz}[4,10], 0.2 \mathrm{~Hz}[5], 0.5 \mathrm{~Hz}[11]$ and $1 \mathrm{~Hz}$ [6]. Even though the BCG spectrum probably extends to these low frequencies, slow balance-related motion and respiration artifacts dominate in these bands for standing BCG recordings. Besides, the effect of low-frequency BCG components on the timing of the J peak may be irrelevant as the peak is a high-frequency feature of the waveform and hence most of its spectrum energy is expected to be on the upper part of the bandwidth. On the other hand, some low-pass cutoff frequencies for BCG used by different research groups are $10 \mathrm{~Hz}[4,16], 15 \mathrm{~Hz}$ [10], $20 \mathrm{~Hz}$ [6] and $25 \mathrm{~Hz}$ [5,11]. No reasons for selecting any particular cutoff frequency seems to have been published but the power density spectrum of a typical BCG obtained from a bathroom scale with the low-pass filter set at $100 \mathrm{~Hz}$ shows that there are significant signal components at least up to $20-25 \mathrm{~Hz}$ (Figure 2). Nevertheless, the J peak is only one of the several waves of the BCG hence its spectrum, which will determine the performance required for filters used to detect it, will not necessarily match that of the entire BCG and is still to be determined.

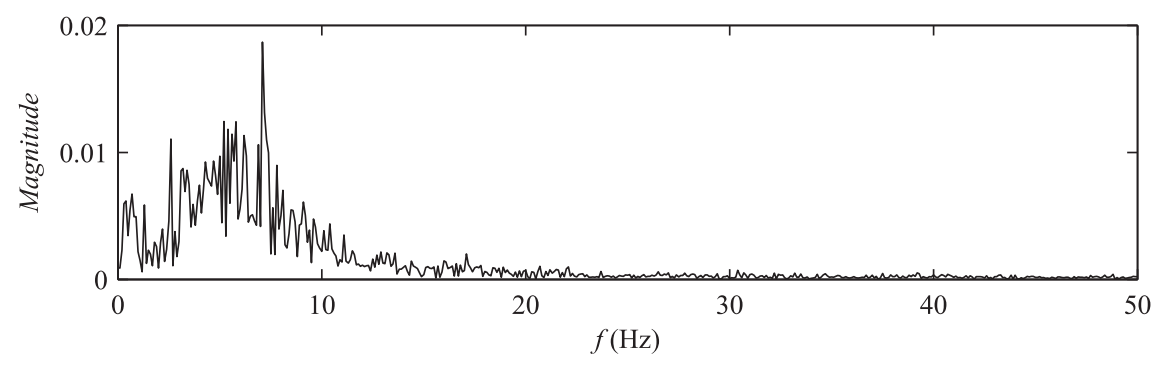

Figure 2. Typical BCG spectrum from a subject that stands on a bathroom scale.

Phase response, sampling frequency, number of bits, noise and interference, which are known sources of uncertainty for ECG systems [13,15], can be expected to affect BCG systems too. Phase response of the transfer function of the analog front-end introduces systematic delays or waveform distortions that may lead to timing displacements of the J peaks. The sampling frequency must fulfill the Nyquist criterion but interpolation techniques used to increase the temporal resolution can introduce timing displacements on the J peaks. The effects of the limited number of bits of the analog-to-digital converter (ADC), noise and power line $(50 / 60 \mathrm{~Hz})$ interference have not been considered in the bibliography either.

This study describes a method to qualitatively estimate the system bandwidth required to accurately time the $\mathrm{J}$ peak of the BCG. Once that bandwidth has been determined, the inherent uncertainty of RJ intervals is estimated and the effects of phase response, sampling frequency, number of bits, noise and interference on the measurement uncertainty are quantified.

\section{Materials and methods}

\subsection{Signal acquisition}

The BCG was acquired from a commercial bathroom scale (Kompernass Balance KH 5510, Kompernass, Germany) by connecting one the two strain gages of each one of its four load cells in a Wheatstone bridge. The bridge output was ac-coupled to a differential amplifier by a fully-differential first-order high-pass filter [17] with cutoff frequency $0.5 \mathrm{~Hz}$, which is high enough to reduce motion artifacts; preliminary tests showed that the frequency components of the J peak were not affected by this filter. The amplifier gain was set to 12,000 and the output was low-pass filtered by a first-order filter with cutoff frequency $100 \mathrm{~Hz}$ hence well above the highest frequency components in Figure 2.

The ECG (lead I) was simultaneously acquired by two pairs of dry stainless steel electrodes mounted on a handle bar. In order to reduce power line interference, one electrode from each pair was connected to ground [18] whereas the other electrode was used to sense the ECG. Electrode signals were ac-coupled to a differential amplifier through a first-order fully-differential high-pass filter with cutoff frequency at $0.5 \mathrm{~Hz}$. Amplifier gain was 1,000 and the output was low-pass filtered by a first-order filter with cutoff frequency at $40 \mathrm{~Hz}$ hence fulfilling the requirements for arrhythmia monitoring [14]. 
Both signals were sampled with a 16 bit data acquisition module (USB $\mu$ DAQ, Eagle Technology, Auckland, New Zealand) at $1 \mathrm{kHz}$ and data was sent to a PC and stored for further analysis. This sampling frequency is high enough according to the Shannon-Nyquist sampling theorem to consider that the signals can be fully recovered with proper interpolation.

The BCG and ECG were simultaneously acquired from 5 volunteers (4 male, 1 female, 24 years to 59 years old, $53 \mathrm{~kg}$ to $68 \mathrm{~kg}$ weight and $1.64 \mathrm{~m}$ to $1.90 \mathrm{~m}$ height), none of which did have any known medical condition. The subjects were selected from the available volunteers in order to include different BCG waveform morphologies in the study, but otherwise they do not represent any particular population group. Subjects were asked to stand still on the scale for $25 \mathrm{~s}$ whereas they held the ECG handle bar. Subjects stood quietly for $60 \mathrm{~s}$ before the experiment in order to stabilize body hemodynamics. A total of 109 full heart cycles were measured.

\subsection{J-peak detection algorithm}

Even though BCG features such as the J peak can be extracted from the signal itself [19], that extraction is easier when a simultaneously recorded ECG is used as a timing reference. We used this approach as the acquisition of this auxiliary signal is easy because the final aim is to measure the RJ interval, which needs the ECG in any case. A simplified version of the Pan-Tompkins algorithm [20] was used to detect R peaks in the ECG signal and absolute maxima of the BCG segments between $150 \mathrm{~ms}$ and $350 \mathrm{~ms}$ after R peaks were annotated as J peaks. Signal processing was performed offline with Matlab ${ }^{\circledR}$.

\subsection{J peak timing bandwidth}

To determine how over-filtering can distort the $\mathrm{J}$ peak, the original records were filtered at different low-pass cutoff frequencies inside the range where we expected to found the upper bandwidth limit, from $7.5 \mathrm{~Hz}$ to $40 \mathrm{~Hz}$ at $2.5 \mathrm{~Hz}$ steps, using a digital $8^{\text {th }}$ order Butterworth low-pass filter, applied forward and reverse to avoid phase response effect, as a good approximation to an ideal filter. The timing of the $\mathrm{J}$ peaks was measured on the resulting samples and for each heartbeat $i$ the displacement of the $\mathrm{J}$ peak $\Delta t_{i}$ on recordings with consecutive frequency cutoffs was measured, as shown in Figure 3..

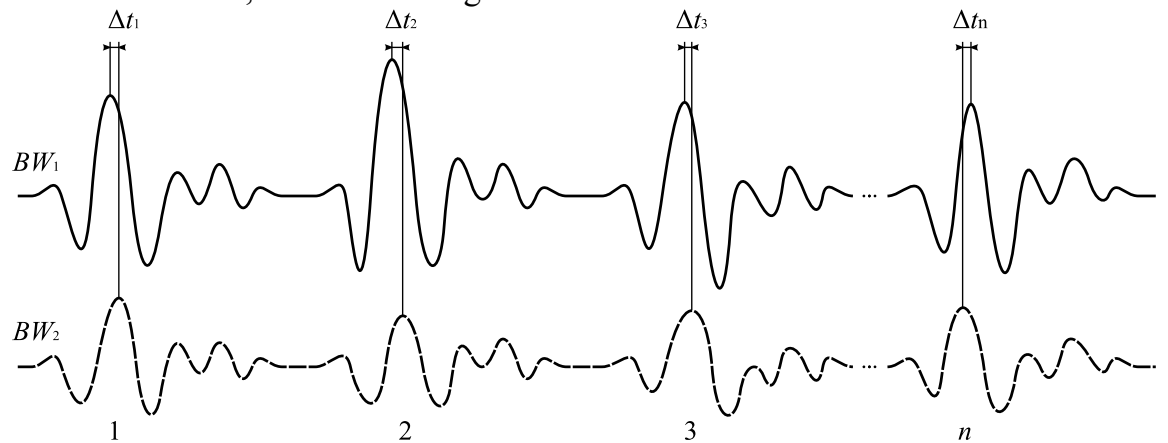

Figure 3. BCG traces obtained with bandwidths $\mathrm{BW}_{1}$ and $\mathrm{BW}_{2}$, and time displacements $\Delta t$ for each heartbeat from 1 to $n$.

The mean $E(\Delta t)$ and the standard deviation $\sigma(\Delta t)$ of the relative displacements for every pair of peaks recorded with consecutive cutoff frequencies were analyzed. Both parameters are expected to change when at least part of the spectrum of J peak is affected by over-filtering. On the one hand, the filter can cause a systematic error in the $\mathrm{J}$ peak time measurement due to a distortion of the J-peak shape. Further, over-filtering can also flatten the peak thus making it more difficult for the detection algorithm to distinguish the true maximum from its neighbor points, hence increasing the uncertainty of measurement.

The same method was used to determine the lower bandwidth limit, but in this case the almost-ideal low-pass filter described above was replaced by an $8^{\text {th }}$ order Butterworth high-pass filter, applied forward and reverse. The frequencies tested were inside the range where we expected to found the limit, from $0.5 \mathrm{~Hz} \mathrm{to} 4 \mathrm{~Hz}$, at $0.25 \mathrm{~Hz}$ steps.

\subsection{Inherent uncertainty of the RJ interval}

Many applications that rely on $\mathrm{J}$ peak timing are oriented to measuring $\mathrm{RJ}$ interval changes induced by paced respiration or slower maneuvers, such as the Valsalva maneuver. Nevertheless, the resolution when tracking these changes is likely to be limited by faster intrinsic fluctuations of the RJ interval also of physiological origin. This inherent uncertainty was quantified from the fluctuations of the RJ interval above $0.5 \mathrm{~Hz}$ because typical fluctuations induced by maneuvers, respiration or hemodynamic regulation have lower frequency. In order to that, the original wide-band BCG signals were first filtered by a digital $8^{\text {th }}$ order Butterworth band-pass filter set between $1 \mathrm{~Hz}$ and $25 \mathrm{~Hz}$ applied forward and reverse, in accordance with the results obtained from the 
bandwidth measurement method described above. This filtering reduced possible effects of noise, interference and motion artifacts in the experiments. After that, the RJ interval was high-pass filtered by a $2^{\text {nd }}$ order Butterworth filter with corner frequency set at $0.5 \mathrm{~Hz}$, which is high enough to suppress the effect of most hemodynamic changes induced by even the fastest respiration rates and then the remaining fluctuations were quantified.

\subsection{Effect of phase response, sampling frequency, number of bits, noise and interference}

To determine the effect of these parameters on the $\mathrm{J}$ peak time measurement, the original wide-band $\mathrm{BCG}$ signals were band-pass filtered in accordance with the bandwidth estimation method as explained above in order to be used as references in the following experiments.

The effect of phase response was quantified by filtering the reference signals by some simulated filters that have been used or could be used in analog front-ends for BCG acquisition: $1^{\text {st }}$ order [4,11], $2^{\text {nd }}$ order Butterworth [10] and $2^{\text {nd }}$ order Bessel. The timing displacement $\Delta t_{i}$ of each J peak $i$ on the filtered signals with respect to the reference was measured and, as the effect of phase response is systematic, only $E(\Delta t)$ was analyzed. High-pass filters were tested in the range from 0.05 to $1 \mathrm{~Hz}$ at $0.25 \mathrm{~Hz}$ steps, and low-pass filters were tested in the range from $25 \mathrm{~Hz}$ to $100 \mathrm{~Hz}$ at $5 \mathrm{~Hz}$ steps.

The sampling frequency effect was quantified by downsampling the reference signals at several factors, from 2 to 20 , which is the maximum allowed by the Nyquist criterion. After that, signals were resampled to $1 \mathrm{kHz}$ by using two common interpolation algorithms: linear and cubic spline. The timing displacement $\Delta t_{i}$ of each J peak $i$ with respect to the timing on the reference records was measured and, as the effect of sampling frequency and interpolation are expected to be random with zero mean, only $\sigma(\Delta t)$ was calculated.

The effect of quantization was measured by digitally decreasing the number of bits of the original signals. The timing displacement $\Delta t_{i}$ of each $\mathrm{J}$ peak $i$ with respect to the timing in the reference signal was measured for different numbers of bits, from 1 to 14 in 1 bit steps. As the effect of quantization is expected to be random with zero mean, only $\sigma(\Delta t)$ was analyzed.

Noise effects were studied by adding different levels of white Gaussian noise to the reference samples. For each noise level, the timing displacement $\Delta t_{i}$ of every J peak $i$ with respect to the timing on the reference records was measured. As noise is a random variable with zero mean, only $\sigma(\Delta t)$ was analyzed.

The same procedure was repeated by adding a $50 \mathrm{~Hz}$ sine wave instead of white Gaussian noise in order to analyze the effect of power line interference.

\section{Results and Discussion}

\subsection{J peak timing bandwidth}

Figure 4 shows the results for the estimation of the upper limit of the BCG bandwidth. $E(\Delta t)$ was almost zero above $22.5 \mathrm{~Hz}$ and $\sigma(\Delta t)$ was nearly constant above that point, hence pointing that higher frequencies had no effect on the timing of the J peak.
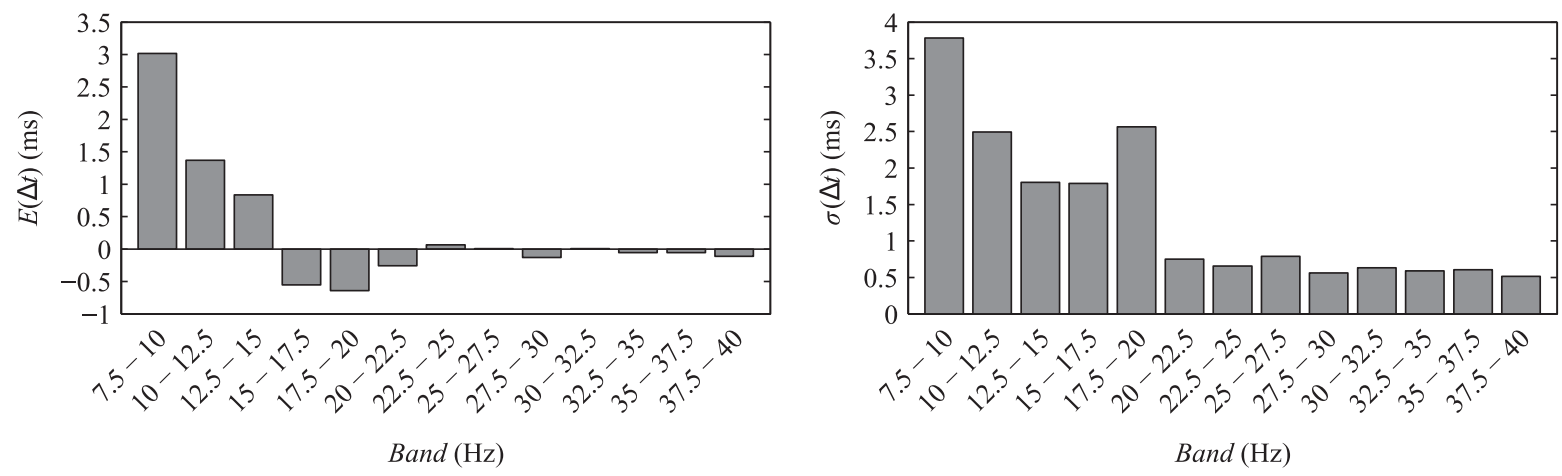

Figure 4. Relative effect of the low-pass cutoff frequency on the timing of the J peak.

Figure 5 shows the results for the estimation for the lower limit of the BCG bandwidth. $E(\Delta t)$ was almost zero below $1.5 \mathrm{~Hz}$ and $\sigma(\Delta t)$ was nearly constant below that point hence pointing out that lower frequencies have no effect on the timing of the J peak. 

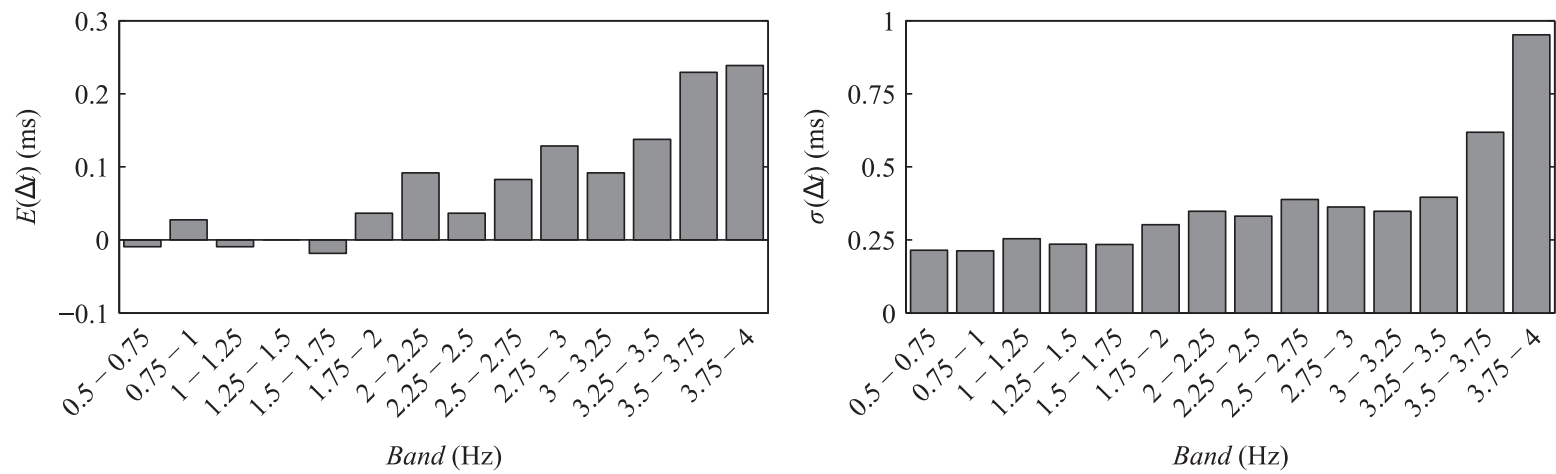

Figure 5. Relative effect of the high-pass cutoff frequency on the timing of the J peak.

The results from the bandwidth estimation method show that the higher frequency bands of the BCG spectrum (Figure 2) are significant on the timing of the J peak hence they should not be filtered out. Quite on the contrary, low frequency components have negligible effect on J peak timing and the high-pass cutoff frequency can be set higher than usual in BCG acquisition systems designed for other purposes. As a consequence, motion artifacts that typically contribute low frequency components can be filtered out hence improving SNR.

\subsection{Inherent uncertainty of the RJ interval}

Figure 6 shows the uncertainty of RJ interval in a histogram. Measured values ranged from $-4 \mathrm{~ms}$ to $3 \mathrm{~ms}$ and their standard deviation was below $2 \mathrm{~ms}$. Therefore, the inherent uncertainty of RJ intervals can be considered to be around $\pm 2 \mathrm{~ms}$. To exclude any possible influence of the original sampling frequency $(1 \mathrm{kHz})$ on this evaluation, the same analysis were repeated after interpolating the signals by a factor 10 to increase time resolution and the same results were obtained.

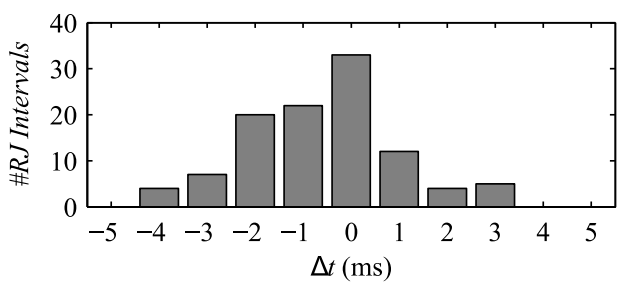

Figure 6. Distribution of the RJ interval uncertainty.

\subsection{Effect of phase response, sampling frequency, number of bits, noise and interference}

Figure 7 shows $E(\Delta t)$ for timing displacements due to the system response of (simulated) common analog filters. At $22.5 \mathrm{~Hz}$, which is the minimal acceptable low-pass cutoff frequency according to the bandwidth estimation above, the minimal mean displacement was that of the first-order filter $(6 \mathrm{~ms})$. This systematic delay increased with increasing filter order and the use of a linear phase (Bessel) filter did not significantly improve it. The minimal mean displacement at the maximum high-pass frequency cutoff determined by the bandwidth estimation method, $2.5 \mathrm{~Hz}$, was that of the first-order filter $(-8 \mathrm{~ms})$. The same as for the low-pass filters, $E(\Delta t)$ increased with filter order and Bessel filters did not significantly improve the results. These timing displacements are larger than the intrinsic uncertainty hence their effect should be considered when absolute RJ interval values are reported.
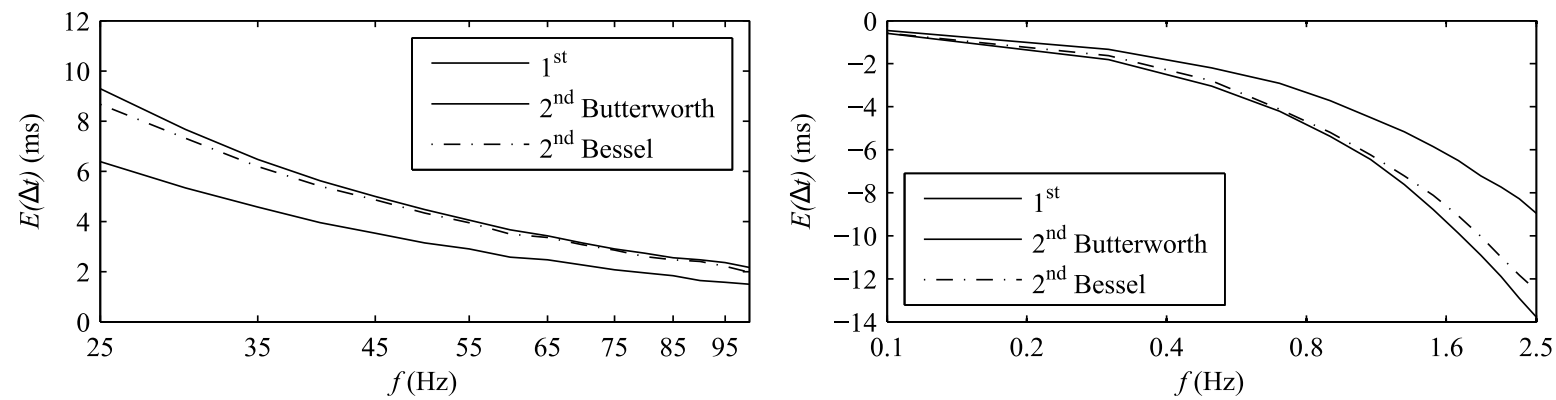

Figure 7. Effect of analog phase response on the timing of the J peak for low-pass (left) and high-pass (right) filters. 
Figure 8 shows $\sigma(\Delta t)$ for different sampling frequencies and interpolation methods. J peaks did not suffer any displacement when the undersampling factor was equal or lower than 6 , which means a sampling frequency of $166 \mathrm{~Hz}$. For higher undersampling, cubic spline interpolation did not introduce more than $2 \mathrm{~ms}$ displacement hence the signal can be sampled at the limit set by the Nyquist theorem (about $50 \mathrm{~Hz}$ ). Linear interpolation performed worse and the displacement introduced was higher than $2 \mathrm{~ms}$ for undersampling factors higher than 7 $(140 \mathrm{~Hz})$. Therefore, if computational resources are available the sampling frequency can be largely reduced.

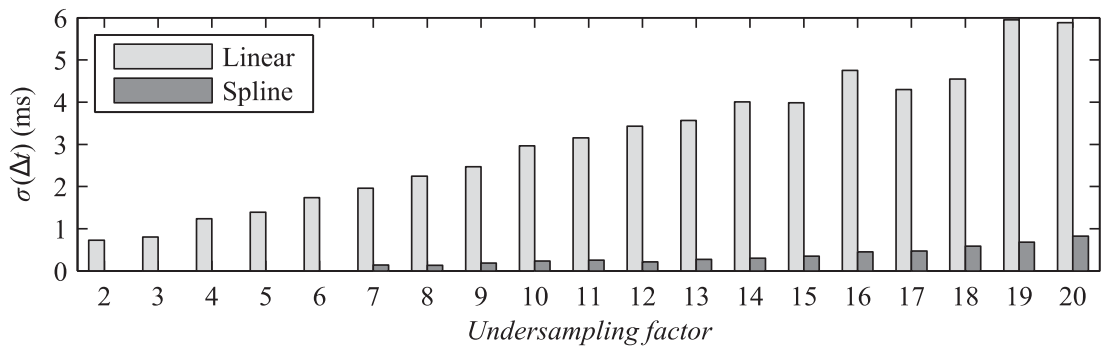

Figure 8. Effect of sampling frequency and interpolation on the timing of the $\mathrm{J}$ peak.

Figure 9 shows the effect of the number of bits on $\sigma(\Delta t)$. Above $N>5$ no significant uncertainty reduction is achieved hence most commercially available data acquisition systems can be used.

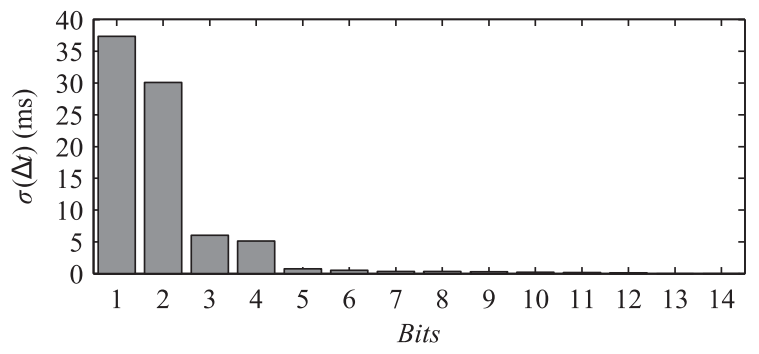

Figure 9. Effect of the number of bits of the ADC on the timing of the J peak.

Figure 10 shows $\sigma(\Delta t)$ as a result of noise and power line interference. The minimum signal-to-noise ratio (SNR) required to achieve $\sigma(\Delta t)<2 \mathrm{~ms}$ was $40 \mathrm{~dB}$ and the minimum signal-to-interference ratio (SIR) was $3 \mathrm{~dB}$. Even though it may seem that the effect of noise is far worse than that from power line interference, it should be noticed that the results strongly dependent on the J-peak timing algorithm. As noise increases, the highest peak in the signal may be located anywhere inside the $200 \mathrm{~ms}$ window after the R peak of the ECG, but the maximal J-peak displacement due to interference will be less than half the period of the interfering signal, $10 \mathrm{~ms}$. Different window lengths or more complex algorithms could yield slightly different results but, in any case, the effect of low SIR will be limited whereas the effect of noise will not.
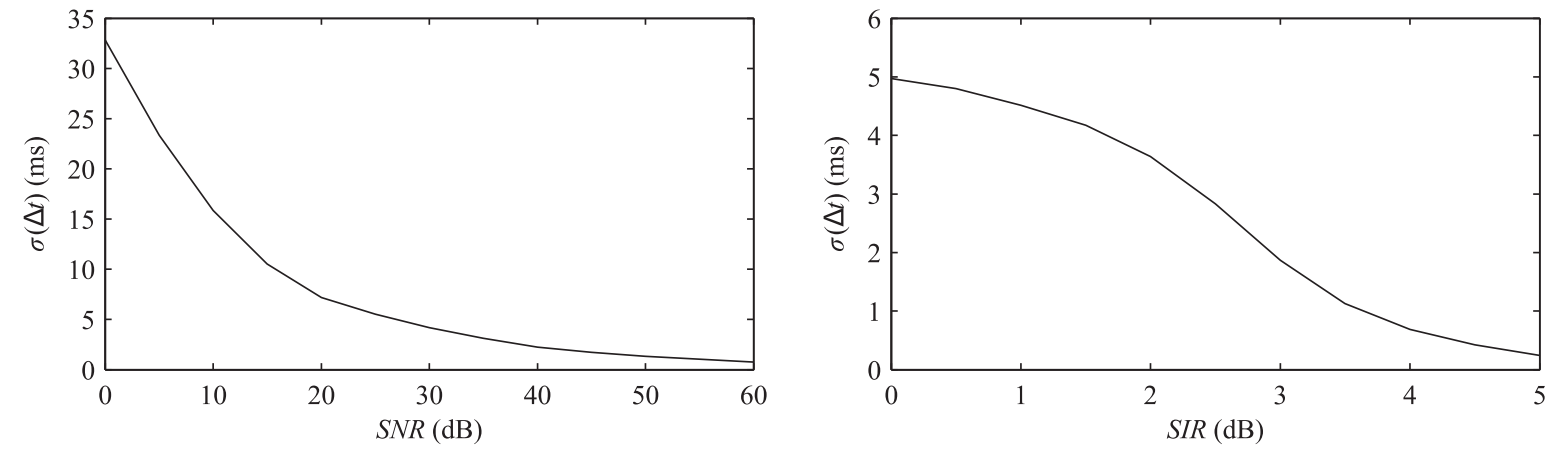

Figure 10. Effect of noise (right) and interference (left) on the timing of the J peak.

\section{Conclusions}

There is currently strong interest on the capabilities of the analysis of the J peak of the BCG to assess hemodynamic parameters but results from different research groups cannot be compared unless minimal performance standards for BCG systems are agreed upon. In this study, we have determined that the minimal system bandwidth to measure the timing of the J-peak of the BCG is from $1.5 \mathrm{~Hz}$ to $22.5 \mathrm{~Hz}$. We have also estimated that the inherent uncertainty in RJ-peak timing is $\pm 2 \mathrm{~ms}$, which establishes a limit for the effects of 
phase response, sampling frequency, number of bits, noise, and power line interference. The high-pass and lowpass cutoff frequencies and filter order to determine the bandwidth cannot be designed by considering SNR or interference rejection criteria only. Timing displacements because of phase response last more than $2 \mathrm{~ms}$ hence its effect should be considered when absolute time values are reported. Interpolation techniques can be used to decrease the sampling frequency without increasing the uncertainty. Cubic spline interpolation offers very good performance but linear interpolation, which is easier to implement, yields acceptable results. ADC resolution better than 5 bits has no significant benefit. SNR higher than $40 \mathrm{~dB}$ and SIR higher than $3 \mathrm{~dB}$ should be achieved to ensure uncertainties below $\pm 2 \mathrm{~ms}$.

These results show that some features of the systems used in previous studies can be downgraded without significantly affecting the measurement, which is important for low-cost systems to be used in broad population groups. At the same line, the minimum bandwidth found is also interesting because it implies that motion artifacts, which are one of the most important problems in in-home BCG measurements, can be filtered out without affecting the measurement. Further studies should be performed in a larger population group to confirm the results above or to improve them, which in any case reinforces the need for the standardization of BCG data acquisition systems intended for measurement of time intervals.

\section{Acknowledgments}

This study has received funding from the Spanish Ministry of Science and Innovation and the European Fund for Regional development (contract TEC2009-13022). The same ministry supports Joan Gomez-Clapers under grant agreement BES-2010-032893. The authors would like to thank the Castelldefels School of Telecommunications and Aerospace Engineering (EETAC-UPC, BarcelonaTech) for its research facilities, Mr. F. Lopez for his technical support and all the volunteers for their patience and valuable collaboration.

\section{References}

[1] P.N. Wells, "Can technology truly reduce healthcare costs?” IEEE Eng Med Biol Mag., vol. 22(1), pp. 2025, 2003.

[2] R.S. Gubner, M. Rodstein, and H.E. Ungerleider, "Ballistocardiography: An appraisal of technic, physiologic principles and clinical value," Circulation, vol. 7 (2), pp. 268-296, 1953.

[3] L. Giovangrandi, O.T. Inan, R.M. Inan, R.M. Wiard, M. Etemadi, and G.T. Kovacs, "Ballistocardiography - a method worth revisting," Conf Proc IEEE Eng Med Biol Soc. 2011, pp. 4279-4282, 2011.

[4] R. Gonzalez-Landaeta, O. Casas, and R. Pallas-Areny, "Heart rate detection from an electronic weighing scale," Physiol Meas., vol. 29 (8), pp. 979-988, 2008.

[5] M. Etemadi, O.T. Inan, L. Giovangrandi, and G.T. Kovacs, "Non-invasive measurement of Valsalvainduced hemodynamic changes on a bathroom scale ballistocardiograph," Conf Proc IEEE Eng Med Biol Soc. 2008, pp. 674-677, 2008.

[6] J. Shin, K.M. Lee, and K.S. Park, "Non-constrained monitoring of systolic blood pressure on a weighing scale,” Physiol Meas., vol. 30 (7), pp. 679-693, 2009.

[7] T. Koivistoinen, S. Junnila, A. Värri, and T. Kööbi, "A new method for measuring the ballistocardiogram using EMFi sensors in a normal chair,” Conf Proc IEEE Eng Med Biol Soc. 2004, pp. 2026-2029, 2004.

[8] J. Paalasmaa, M. Waris, H. Toivonen, L. Leppäkorpi, and M. Partinen, "Unobtrusive online monitoring of sleep at home," Conf Proc IEEE Eng Med Biol Soc. 2012, pp. 3784-3788, 2012.

[9] P. Pollock, "Ballistocardiography: a clinical review," Can Med Assoc J., vol. 76(9), pp. 778-783, 1957.

[10] M. Etemadi, O.T. Inan, L. Giovangrandi, and G.T. Kovacs, "Rapid assessment of cardiac contractility on a home bathroom scale,” IEEE Trans Inf Technol Biomed., vol. 15(6), pp. 864-869, 2011.

[11] R. Casanella, J. Gomez-Clapers, and R. Pallas-Areny, "On time interval measurements using BCG," Proc IEEE Eng Med Biol Soc Conf. 2012, pp. 5034-5037, 2012.

[12] BIPM, IEC, IFCC, ILAC, ISO, IUPAC, IUPAP and OIML, JCGM 100:2008 Evaluation of Measurement Data - Guide to the Expression of Uncertainty in Measurement (GUM 1995 with minor corrections). Joint Committee for Guides in Metrology.

[13] J.J. Bailey, A.S. Berson, A.Jr. Garson, L.G. Horan, P.W. Macfarlane, D.W. Mortara, and C. Zywietz, "Recommendations for standardization an specifications in automated electrocardiography: bandwidth and digital signal processing. A report for health professionals by an ad hoc writing group of the Comittee on Electrocardiography and Cardiac Electrophysiology of the Council on Clinical Cardiology, American Heart Association," Circulation, vol. 81(2), pp. 730-739, 1990.

[14] AAMI/IEC, 60601-2-47:2012 Medical electrical equipment - Part 2-47: Particular requirements for the safety, including essential performance, of ambulatory electrocardiographic systems, Association for the Advancement of Medical Instrumentation (AAMI). 
[15] J. Gomez-Clapers, A. Serra-Rocamora, R. Casanella, and R. Pallas-Areny, "Uncertainty factors in timeinterval measurements in BCG," Proc. of the $19^{\text {th }}$ IMEKO TC4 Symposium on Measurements of Electrical Quantities, pp. 395-399, 2013.

[16] O.T. Inan, "Brief history of the BCG," Novel technologies for cardiovascular monitoring using ballistocardiography and electrocardiography, pp. 12-13, Ed. ProQuest, UMI dissertation publish, 2011.

[17] E. M. Spinelli, R. Pallas-Areny and M. A. Mayosky, "AC-coupled front-end for biopotential measurements," IEEE Trans Biomed Eng., vol. 50(3), pp. 391-395, 2003.

[18] D. Diaz, O. Casas, and R. Pallas-Areny, "Interference reduction in ECG recordings using a dual ground electrode," Proc. of the $19^{\text {th }}$ IMEKO World Congress on Fundamental and Applied Metrology, pp. 16951700, 2009.

[19] A. Akhbardeh, B. Kaminska, and K. Tavakolian, "BSeg++: a modified segmentation method for Ballistocardiogram cycle extraction," Conf Proc IEEE Eng Med Biol Soc. 2007, pp. 1896-1899, 2007.

[20] J. Pan, and W.J. Tompkins, “A real-time QRS detection algorithm," IEEE Trans Biomed Eng., vol. 32(3), pp. 230-236, 1985. 